\title{
Prevention and control measures of the major cold- region diseases (hypertension) in China
}

\author{
Zhenwei Pan ${ }^{1}$, Yong Zhang ${ }^{1}$, Tengfei Pan ${ }^{1}$, Haihai Liang ${ }^{1}$, Baofeng Yang ${ }^{1,2^{*}}$
}

\begin{abstract}
Hypertension is the most common cardiovascular condition in clinical practice and a major risk factor for stroke and cardiovascular events. There are more than 270 million hypertension patients in China, and the prevalence of hypertension in the high-latitude cold areas is significantly higher than in the low-latitude warm areas. The unique epidemiological characteristics and risk factors of hypertension in the cold regions of China urge for establishment of the prevention and control system for targeted and more effective management of the condition.
\end{abstract}

\section{Keywords}

hypertension; cold region; prevention; cure

Received O2 February 2021 accepted 7 March 2021

\section{Introduction}

Hypertension is a vascular disease of rather detrimental consequences with a high rate of death and disability and has become one of the most common health conditions in the world. According to The Report on Nutrition and Chronic Diseases of Chinese Residents (2015), the prevalence of hypertension among residents aged 18 and above was $25.2 \%$, but the awareness rate, treatment rate and control rate were only $46.5 \%, 41.1 \%$ and $13.8 \%$, respectively, addressing the critical situation of hypertension prevention and control in China. China covers a vast territory of different climate zones with different prevalence rates of hypertension. Northern China is within the cold region (with an average temperature in January below $-8^{\circ} \mathrm{C}$ ) spanning 4 6-months frigid weather conditions. People's eating habits and lifestyle in northern China are overall more calorie- and fat-enriched and less physically active than in southern China. Residents living in cold areas of high latitude are constantly challenged by higher risk and prevalence of hypertension than those in warm areas of low latitude. Therefore, implementing strategy and measures that lock hypertension under effective prevention and control in cold regions is of great significance for promoting health status, quality of life, and even the development of the whole society and economy.

\section{Epidemic, harm and prevention strategies and measures of hypertension}

Hypertension is a chronic disease with elevated arterial blood
1 Department of Pharmacology (The Key Laboratory of Cardiovascular Research, Ministry of Education) at College of Pharmacy, Harbin Medical University, Harbin 150086, China

2 Research Unit of Noninfectious Chronic Diseases in Frigid Zone, Chinese Academy of Medical Sciences, China, 2019RU070

*Corresponding author Prof. Baofeng Yang, E-mail: yangbf@ems.hrbmu.edu.cn pressure, defined by Chinese criteria as systolic blood pressure $\geq 140 \mathrm{mmHg}$ and/or diastolic blood pressure $\geq 90 \mathrm{mmHg}$ at resting state without the use of antihypertensive drugs[1]. It is one of the most harmful and prevalent diseases of the circulatory system[2]. This condition can cause a series of clinical symptoms by adversely affecting the function of many important internal organs, especially the heart, brain, and kidney, eventually leading to organ failure.

In 2010 , about $31.1 \%$ of adults (1.39 billion people) worldwide had hypertension[3]. It is estimated that by 2025 , global adults with hypertension will reach 1.56 billion[4]. The incidence and mortality of hypertension are exceptionally high in China. The latest hypertension epidemiological survey of community population shows that the prevalence rate of hypertension in 35 75 years old Chinese population is $44.7 \%[5]$. Another survey publicized in 2019 revealed an astounding number of 245 million hypertensive Chinese adults over the age of $18[6]$.

Hypertension is regarded as a "stealth killer" characterized by high morbidity, high prevalence, and high complication rates as well as its concealed and asymptomatic progression. Prior to the appearance of symptoms, hypertension often has already caused severe damages to multiple organs (including heart, brain, and kidney) as an independent risk factor of heart failure[7], coronary heart disease[8], kidney, and peripheral vascular diseases (atherosclerosis, stroke, etc.)[9]. If remained uncontrolled and untreated timely, it will lead to a variety of complications, such as cardiovascular, brain and kidney complications, rendering patients in health-endangering or even live-threatening situations. 
Many factors are involved in the pathogenesis and pathological progression of hypertension. Individual factors such as heredity[10], age, diet habit[11] and lifestyle[12] are closely related to the incidence of hypertension. Likewise, environmental factors also affect the prevalence of hypertension. The results from surveys and statistical analyses revealed that the populations with high levels of education have a significantly lower risk of hypertension in China. Yet, no significant correlation exists between hypertension and gender or income. Obesity, family history, diabetes, overweight, waist-to-hip ratio, and high salt intake all are independent risk factors of hypertension. Therefore, the key to prevent and treat hypertension is adopting healthy lifestyle to minimize the impacts of these risk factors.

International guidelines to general hypertension prevention and treatment are formulated for the whole population, highrisk groups and hypertension patients, respectively[13, 14]. Combination of prevention and treatment is the fundamental strategy for hypertension control of the general population, as well as the high-risk groups and hypertensive patients. The strategy for the whole population is primarily national health education, medical knowledge popularization, adopting healthy lifestyle and participating in health management. Additionally, taking full use of the existing healthcare service network to boost the participation of entire communities in the prevention and treatment of hypertension in the manner of close cooperation among multiple health sectors and the government at all levels[15]. The strategy for the high-risk groups is to provide them with intensive health education, guide them to adopt healthy lifestyle while dropping unhealthy lifestyle and behavior, and urge them to quit smoking, restrict alcohol consumption, limit salt intake, and go on a diet and do regular physical exercise[16-17]. At the same time, early identification and control of risk factors of hypertension, prevention and delay of the development of hypertension and associated complications are important. The measures for hypertension patients are primordial prevention including early detection, early diagnosis and early treatment, standardized management and monitoring, and creation of personal health records with connection to community's routine diagnosis and treatment information system for uninterrupted healthcare services[18-19]. The international guidelines for hypertension prevention and treatment stress to abandon unhealthy lifestyles, limit daily sodium intake, persist in weight control and regular exercise, quit smoking, and restrict alcohol consumption in conjunction with community intervention, health education and psychological intervention[20].

\section{Impact of chilliness on the prevalence, harm, and prevention of hypertension}

A large number of epidemiological studies have proved that cold environment increases blood pressure[21-23]. Meanwhile, many studies have found that the incidence rate and mortality rate of cardiovascular diseases in high latitude and alpine regions are higher in winter[24]. A study was conducted to compare the changes of blood pressure in different seasons in 2051 adults. The results show that the mean systolic/diastolic blood pressure (SBP/DBP) in the hot season was $126.8 \pm 0.8 / 82.2 \pm 0.8 \mathrm{mmHg}$, and the mean SBP/DBP in the cold season was $130.3 \pm 0.6 / 84.4 \pm 0.7$ $\mathrm{mmHg}$. Subgroup analysis revealed that the blood pressure of three different groups (normal BP, untreated hypertension patients and treated hypertension patients) was all significantly higher in the cold season than in the hot season $(\mathrm{P}<0.05)$. A prospective clinical study in 500,000 residents from 10 provinces/cities in China revealed that SBP and DBP are $10 \mathrm{mmHg}$ and $4.2 \mathrm{mmHg}$ higher, respectively, in the cold season (December to February the following year) than in the hot season (June to August) $(P<0.05)$ [25]. In addition, the incidence and severity of cerebrovascular diseases as hypertension complications are also closely related to the cold climate[26].

The relationship between blood pressure and different seasons, as well as indoor and outdoor temperatures was systematically analyzed in the China Chronic Disease Cohort Study[25]. A total of 506,673 urban and rural residents aged 35 to 79 years from 10 different areas were surveyed and analyzed. The data revealed a $10-\mathrm{mmHg}$ difference in averaged SBP between the summer (June to August) and the winter (December to February). There is a negative correlation between SBP and outdoor temperature above $5^{\circ} \mathrm{C}$ in all 10 regions with a $5.7-\mathrm{mmHg}$ increment for every $10^{\circ} \mathrm{C}$ decrement in outdoor temperature[25]. The correlation of SBP with the outdoor temperature is more significant in older adults and those with a lower body mass index. At lower temperatures, the correlation is less pronounced in centrally heated homes. Increased blood pressure upon exposure to cold temperatures is called cold-stress hypertension (CSH). The main susceptible population is the elderly and people with a low body mass index. The clinical characteristic is that blood pressure rises in daytime and drops at night, and such fluctuation is more common in patients with $\mathrm{H}$-type hypertension (hypertension coupled with high blood homocysteine). When the weather becomes cold, especially when the temperature drops sharply, the sympathetic nervous system and the RAS system are over-activated to raise blood pressure, leading to $\mathrm{CSH}[27-28]$, with increased incidence and mortality of various cardiovascular and cerebrovascular complications[29-30].

Cold weather or environment has great influence on the diagnosis and treatment of hypertension. Cold exposure can cause profound changes in blood pressure differences between young and elder, men and women, and fat and thin people. Hence, environmental temperature must be taken into account for the diagnosis of hypertension, and so must the treatment of hypertension. In 
addition, the antihypertensive regimen should be re-adjusted in the summer according to the ambient temperature. Moreover, selection of antihypertensive drugs that could better counter the pathogenesis of cold-induced hypertension should become a guideline for clinical practice. For example, RAS inhibitors or vessel dilators may be one of the appropriate antihypertensive agents in cold areas[31].

At present, there has not been any specific guidelines and recommendations on the prevention and treatment standards of cold-induced hypertension in China and other countries. Yet, different intervention measures are being taken in different temperature environments to reduce the adverse effects of high or low blood pressure and the risk of cardiovascular and cerebrovascular complications and/or comorbidities, especially for the elderly. For example, systolic and diastolic blood pressure levels in cold season can be significantly reduced by improving heating and dwelling insulation conditions and reducing exposure time to cold environment. Being on healthy diet and abandoning unhealthy dietary habits can also reduce the risk of hypertension; hypertension patients should take on light diet[32] with appropriate proportions of coarse and find food and of meat and vegetables. In addition, exposure to sunlight, appropriate vitamin D supplement, and proper intensity of physical activities are all helpful for the prevention of cold-induced hypertension.

It has been suggested that regular 24-hour dynamic blood pressure monitoring or blood pressure self-testing should be considered for accurate assessment of blood pressure fluctuations in cold seasons. On the other hand, timely and effective antihypertensive treatment should be taken with standardized and individualized regimen to reduce hypertension-related cardiovascular and cerebrovascular complications and improve quality of life.

\section{Prevalence and prevention of hypertension in China's cold regions}

Cold regions refer to the geographic areas where the average temperature in January is below $-8^{\circ} \mathrm{C}$ or the average daily high temperature is below $0^{\circ} \mathrm{C}$ for a long period within a year with precipitation often in the form of snow, shorter sunlight duration, and distinct four seasons. China's cold regions account for more than half of the country's territory, and the number of people living in cold regions exceeds 200 million. They include three northeastern provinces of China, Inner Mongolia, and some areas in northwest and southwest China, all within the regions with relatively high latitude and altitude in China.

According to a research publicized in 2018, the average prevalence of hypertension was $29.70 \%$ in cold region provinces and $26.30 \%$ in non-cold regions of China[33]. Another research reported that the prevalence of hypertension was the highest in the eastern region (32.6\%), followed by the northeast region $(31.8 \%)$, and the control rate of hypertension in southwest China (4.8\%) and northeast China (5.9\%) was very poor[34]. In Heilongjiang, a northeast province of China, the prevalence of hypertension was up to $30.76 \%$ among residents over 18 years old in 2015[35].

The analysis and comparison of the main risk factors of hypertension between cold and non-cold regions uncovered that besides cold weather, other risk factors, such as the rates of high blood triglyceride, diabetes, overweight and obesity, and excessive salt intake were also higher in the nine cold-region provinces than in the non-cold regions. In contrast, the rates of insufficient vegetable and fruit intake and physical exercise were lower in cold regions than in the non-cold regions. Attributable risk analysis revealed that $48.86 \%$ of hypertension in cold regions of China was related to overweight and obesity, $32.45 \%$ to high salt intake, $9.78 \%$ to diabetes, $9.57 \%$ to drinking and $6.97 \%$ to smoking.

The results of the questionnaire survey provided by the Centers for Disease Control and Prevention of all provinces (districts) showed that the average awareness rate, treatment rate and control rate of hypertension patients in nine cold-region provinces were lower than in non-cold region provinces and the national average[33].

According to the survey on the implementation of prevention and control measures, eight provinces/autonomous regions in the cold regions have been conducting regular special investigations on chronic diseases and risk factors and have established demonstration areas for comprehensive prevention and control of chronic diseases. However, half of the provinces/autonomous regions have not established comprehensive monitoring sites for chronic diseases, and two provinces/autonomous regions have not established the resident health record system. Eight coldregion provinces/autonomous regions have carried out regular dissemination on hypertension, and seven provinces/autonomous regions have not carried out salt reduction act. Identification of hypertension patients in eight cold provinces/regions was mainly achieved via the blood pressure measurement system for initial diagnosis in the outpatient department, followed by hypertension measurement stations, community visits, free diagnosis, and screening of high-risk groups. Eight provinces/ autonomous regions in the cold region have established hypertension prevention and control networks and implemented national basic public health programs for chronic diseases. Five provinces/autonomous regions have summarized and shared their hypertension prevention and control experience. 
The recommendations for future hypertension prevention and treatment from eight cold-region provinces/ autonomous regions mainly include: (a) to improve the essential hypertension medication system; (b) to provide intensive technical training programs to grassroots professionals for improving the treatment and management of hypertension; (c) to accelerate the construction of chronic disease prevention and treatment management platforms and the informatization process of hypertension health records. Additional suggestions are to increase government financial investment, improve two-way referral of hypertension patients, popularize appropriate technologies, and explore hypertension prevention and control measures and technical solutions suitable for the poor areas in western China.

\section{Experience, advantages, disadvantages and suggestions on the prevention and treatment of hypertension in China}

The central government of China plays a leading role in formulating appropriate policies and regulations and making long-term plans for optimizing the management of hypertension nationwide. Academic bodies and medical institutions give full play to their professional expertise, formulate guidelines, provide training and guidance to the public, and facilitate the standardization of hypertension diagnosis and treatment. The grassroot community implements the comprehensive hypertension prevention and treatment model based on China's national conditions and apply the hypertension prevention and treatment policy, measures, and experience to the community practice. Mass media is actively disseminating the healthy lifestyle and popularizing the hypertension prevention knowledge to enhance the public health consciousness and the healthcare level. Through the joint efforts of the whole society, the prevention and treatment of hypertension in China have made great achievements and accumulated rich and valuable experience in the prevention and treatment of hypertension, which is widely recognized by international counterparts. These prevention and treatment experiences are herein outlined. (a) The government plays a leading role in promoting the optimal management of hypertension in the whole society. (b) Academic groups and medical institutions give full play to their professional advantages to promote the standardization of hypertension diagnosis and treatment. (c) Grassroot communities actively implement the comprehensive prevention and treatment model for hypertension which is suitable for China's national circumstances. (d) Mass media actively disseminate healthy lifestyle and popularize the knowledge about hypertension prevention and treatment.

The Chinese model of hypertension prevention and treatment manifests several advantages. (a) The central government of China attaches great importance to chronic disease prevention and control. (b) There exists multi-sectoral cooperation under the guidance of policies and legal protection. (c) There exists a comprehensive cardiovascular disease (including hypertension) prevention and control system jointly formed by the National Center for Disease Control, the Center for Chronic Diseases, and the National Center for Cardiovascular Diseases. (d) The epidemiological studies on hypertension have been constantly becoming more insightful and standardized. (e) Hypertension prevention guidelines and the related technical specifications have been widely implemented.

The deficiencies of the hypertension prevention and treatment in China are manifested in the following aspects. (a) The epidemic trend of hypertension is extremely severe, "three low and one high" (low awareness rate, low treatment rate, low control rate, and high incidence) continues to aggravate, medical resources and technologies are seriously differentiated, and tertiary hospitals are overloaded and overwhelmed. (b) Most hospitals lack hypertensive specialties, which leads to the non-standard fragmented management of hypertensive patients. (c) The family doctor system does not presently exist in China, which negatively impacts the prevention and control of hypertension and other chronic diseases. (d) Lack of perfect professional and technical service organizations and basic health service system and institution. (e) Lack of systematic and operability research on hypertension prevention and control technologies. (f) Insufficient popularization of hypertension diagnosis and treatment technologies. (g) The ability to prevent and control hypertension in cold regions of China is relatively weak.

Recommendations for hypertension prevention and treatment in China are as follows. (a) Establish and consolidate the "government-led, multi-sector cooperative and whole-society participating" working model for chronic disease prevention and treatment. (b) Establish guarantee mechanisms to give full play to the community hypertension management[36]. (c) Pay attention to the popularization of hypertension prevention knowledge, provide education on hypertension prevention knowledge in primary and secondary schools. (d) Set up hypertension specialty in the hospitals with a ranking above level 2 .

The strategies for hypertension prevention and treatment in cold regions of China are suggested as follows. (a) Establish the national key laboratory for chronic disease prevention and treatment and actively carry out scientific research on hypertensionrelated diseases in cold regions. (b) Take corresponding measures to minimize the main risk factors of hypertension in cold areas and achieve precise prevention and control. (c) Reinforce the building up of the capability of hypertension prevention and treatment professional team in cold areas. (d) Incorporate the hypertension prevention and treatment practice into the health poverty alleviation project in cold areas[37]. 


\section{Acknowledgements}

This work was supported by National Natural Science Foundation of China (81730012, 81861128022 to B. Y.), Heilongjiang Touyan Innovation Team Program, and CAMS Innovation Fund for Medical Sciences (CIFMS, 2019-I2M-5-078 to B. Y.).

\section{Conflict of Interest}

Baofeng Yang is the Editor-in-Chief of Frigid Zone Medicine. Zhenwei Pan and Yong Zhang are Editorial Board Members. The article was subject to the journal's standard procedures, with peer review handled independently of this Member and their research groups.

\section{References}

[1] Williams B, Mancia G, Spiering W, et al. 2018 ESC/ESH Guidelines for the management of arterial hypertension. European heart journal, 2018;39(33):3021-3104.

[2] London G, Safar M, Weiss Y, et al. Volume-dependent parameters in essential hypertension. Kidney international, 1977;11(3):204-208.

[3] Mills K, Bundy J, Kelly T, et al. Global Disparities of Hypertension Prevalence and Control: A Systematic Analysis of Population-Based Studies From 90 Countries. Circulation, 2016;134(6):441-450.

[4] Kearney P, Whelton M, Reynolds K, et al. Global burden of hypertension: analysis of worldwide data. Lancet (London, Engla nd),2005;365(9455):217-223.

[5] Lu J, Lu Y, Wang X, et al. Prevalence, awareness, treatment, and control of hypertension in China: data from 1.7 million adults in a population-based screening study (China PEACE Million Persons Project). Lancet (London, England), 2017;390(10112):2549-2558.

[6] China TWCotRoCHDi. Summary of cardiovascular health and disease report 2019 in China. Chinese Circulation Journal, 2020;35(9):833-854.

[7] Tocci G, Sciarretta S, Volpe M. Development of heart failure in recent hypertension trials. Journal of Hypertension,2008;26(7):1477-1486.

[8] Gorelick P, Qureshi S, Farooq M. Management of blood pressure in stroke. International Journal of Cardiology Hypertension, 2019;3:100021.

[9] Gaddam K, Verma A, Thompson M, et al. Hypertension and cardiac failure in its various forms. The Medical clinics of North America,2009;93(3):665-680.

[10] Hung G, Gu D, Wu X, et al. The influence of parental history of hypertension on the prevalence of hypertension and blood pressure in children. Chinese Journal of Hypertension,2002(3):81-84.

[11] Buendia J, Bradlee M, Daniels S, et al.Longitudinal effects of dietary sodium and potassium on blood pressure in adolescent girls. JAMA pediatrics, 2015;169(6):560-568.

[12] Forman J, Stampfer M, Curhan G. Diet and lifestyle risk factors associated with incident hypertension in women. JAMA, 2009;302(4):401411.

[13] Page M. The JNC 8 hypertension guidelines: an in-depth guide. The American Journal of Managed Care, 2014;20:E8.

[14] Polonia J, Martins L, Pinto F, et al.Prevalence, awareness, treatment and control of hypertension and salt intake in Portugal: changes over a decade. The PHYSA study. Journal of Hypertension, 2014;32(6):12111221.

[15] Carey R, Whelton P. Prevention, Detection, Evaluation, and Management of High Blood Pressure in Adults: Synopsis of the 2017 American College of Cardiology/American Heart Association Hypertension Guideline. Annals of Internal Medicine,2018;168(5):351-358.

[16] Kotseva K, De Backer G, De Bacquer D, et al. Lifestyle and impact on cardiovascular risk factor control in coronary patients across 27 countries: Results from the European Society of Cardiology ESC-EORP EUROASPIRE V registry. European Journal of Preventive Cardiology,
2019;26(8):824-835

[17] Krist A, Davidson K, Mangione C, et al. Behavioral Counseling Interventions to Promote a Healthy Diet and Physical Activity for Cardiovascular Disease Prevention in Adults With Cardiovascular Risk Factors: US Preventive Services Task Force Recommendation Statement. JAMA, 2020;324(20):2069-2075.

[18] Smith S, Wallace E, O'Dowd T, et al.Interventions for improving outcomes in patients with multimorbidity in primary care and community settings. The Cochrane Database of Systematic Reviews,2021;1:CD006560.

[19] Chobanian A, Bakris G, Black H, et al. The Seventh Report of the Joint National Committee on Prevention, Detection, Evaluation, and Treatment of High Blood Pressure: the JNC 7 report. JAMA,003;289(19):2560-2572. [20] Mancia G, Fagard R, Narkiewicz K, et al. 2013 ESH/ESC guidelines for the management of arterial hypertension: the Task Force for the Management of Arterial Hypertension of the European Society of Hypertension (ESH) and of the European Society of Cardiology (ESC). European Heart Journal,2013;34(28):2159-2219.

[21] Brennan P, Greenberg G, Miall W, et al. Seasonal variation in arterial blood pressure. British medical journal (Clinical research ed), 1982;285(6346):919-923.

[22] Brook R, Weder A, Rajagopalan S. "Environmental hypertensionology" the effects of environmental factors on blood pressure in clinical practice and research. Journal of Clinical Hypertension (Greenwich, Conn),2011;13(11):836-842.

[23] Matsumoto M, Ishikawa S, Kajii E. Cumulative effects of weather on stroke incidence: a multi-community cohort study in Japan. Journal of Epid emiology,2010;20(2):136-142.

[24] Bhatnagar A. Environmental Determinants of Cardiovascular Disease. Circulation Research, 2017;121(2):162-180.

[25] Lewington S, Li L, Sherliker P, et al. Seasonal variation in blood pressure and its relationship with outdoor temperature in 10 diverse regions of China: the China Kadoorie Biobank. Journal of Hypertensi on,2012;30(7):1383-1391.

[26] Manfredini R, Gallerani M, Portaluppi F, et al. Chronobiological patterns of onset of acute cerebrovascular diseases. Thrombosis Research,1997;88(6):451-463.

[27] Sun Z. Cardiovascular responses to cold exposure. Frontiers in bioscience (Elite edition),2010;2:495-503.

[28] Sun Z, Cade R, Zhang Z, et al.Angiotensinogen gene knockout delays and attenuates cold-induced hypertension. Hypertension (Dallas, Tex : 1979), 2003;41(2):322-327.

[29] Forsdyke D. Summertime dosage-dependent hypersensitivity to an angiotensin II receptor blocker. BMC research notes,2015;8:227.

[30] Hong Y, Kim H, Lim Y, et al.Identification of RAS genotypes that modulate blood pressure change by outdoor temperature. Hypertension research : official journal of the Japanese Society of Hypertensi 
on,2013;36(6):540-545.

[31] Shechtman O, Fregly M, van Bergen P, et al. Prevention of coldinduced increase in blood pressure of rats by captopril. Hypertension (Dallas, Tex : 1979), 1991;17:763-770.

[32] Song $X$. Study on the relationship between dietary nutrition and hypertension. China Health Industry,2014;11(25):22-23.

[33] Tong S, Zhou L, Zhang H, et al. Meta-analysis of the prevalence of hypertension in cold and non-cold areas of China. Chinese Journal of End emiology,2018;37(05):420-425.

[34] Li D, Lv J, Liu F, et al. Hypertension burden and control in mainland China: Analysis of nationwide data 2003-2012. International Journal of
Cardiology,2015;184:637-644.

[35] Yan S C, Zhou X, Jin L, et al. Prevalence, awareness and control of hypertension among adults in Heilongjiang Province in 2015. Chinese Journal of Public Health Management,2019;35(06):749-752.

[36] Li H, Wu Z, Hui X, et al. Impact of local health insurance schemes on primary care management and control of hypertension: a cross-sectional study in Shenzhen, China. BMJ Open. 2019;9(10):e031098.

[37] Zhao Y, Mahal A, Tang S, et al. Effective coverage for hypertension treatment among middle-aged adults and the older population in China, 2011 to 2013: A nationwide longitudinal study. Journal of Global Health. 2020;10(1):010805. 